\title{
Electron Beam Irradiation of Gallium Nitride-on-Silicon Betavoltaics Fabricated with a Triple Mesa Etch
}

\author{
T. Heuser, ${ }^{1,}$ a) M. Braun, ${ }^{1}$ P. Mclntyre, ${ }^{1}$ and D.G. Senesky ${ }^{2,3}$ \\ 1) Department of Materials Science and Engineering, Stanford University, 450 Serra Mall, Stanford, \\ CA 94305 \\ ${ }^{2)}$ Department of Aeronautics and Astronautics, Stanford University, 450 Serra Mall, Stanford, \\ CA 94305 \\ 3) Department of Electrical Engineering, Stanford University, 450 Serra Mall, Stanford, \\ CA 94305
}

(Dated: 27 August 2021)

\begin{abstract}
A process for growing gallium nitride $(\mathrm{GaN})$ vertical p-i-n homojunctions on (111) silicon (Si) substrates using metalorganic chemical vapor deposition (MOCVD) was developed, and a triple mesa etch technique was used to fabricate efficient betavoltaic energy converters. Monte Carlo simulation platform CASINO was used to model beta radiation penetration into GaN to aid device design. The resulting devices were tested under irradiation from a scanning electron microscope (SEM) electron beam (e-beam) tuned to imitate the energies of the ${ }^{63} \mathrm{Ni}$ beta emission spectrum. Based on current-voltage (I-V) measurements taken under e-beam illumination, a maximum open-circuit voltage of $412 \mathrm{mV}$ and a maximum short-circuit current density of $407 \mathrm{nA} / \mathrm{cm}^{2}$ were measured. A high fill factor (FF) of 0.77 and power conversion efficiency of $6.6 \%$ were obtained. Additionally, the proposed triple mesa etch technique used to create these betavoltaics has potential for further use in fabricating many types of electronic devices using a wide variety of material platforms.
\end{abstract}

\section{INTRODUCTION}

Betavoltaic energy converters are semiconductor devices that harvest energy from decaying radioisotopes. Unlike radioisotope thermoelectric generators (RTGs), which harvest heat produced by decaying radioisotopes, betavoltaics convert the energy from ionizing radiation directly into electricity. Originally developed in the mid-1950s, early betavoltaics were silicon ( $\mathrm{Si}$ ) devices powered by ${ }^{147} \mathrm{Pm}$, and were used to power implantable pacemakers! 12

Betavoltaics have significant potential as an extremely versatile power source for electronic devices in situations requiring small size, long operational lifetimes, in which conventional means of power generation are difficult, or in locations where harsh environments (high temperature, radiation-rich, corrosive, etc.) preclude the use of other types of power sources. Examples include remote sensor platforms for space, deep sea, deep underground, biomedical implants, and in-situ structural health monitoring. 3/4

The physics principles behind the operation of betavoltaics are very similar to those of photovoltaics. Both utilize the electric field built up across the depletion region of a semiconductor junction to capture electrons and holes generated by incident energetic particles. However, photovoltaics are powered by light (photons), whereas betavoltaics are powered by beta radiation (electrons). As beta particles travel through the semiconductor, a substantial fraction of their energy is used to create electron-hole pairs (EHPs) along their path.5 ${ }^{\text {Signifi- }}$ cantly, since the energy of beta particles, even those produced by relatively low-energy isotopes, can be orders of magnitude higher than the semiconductor bandgap energy, each incident particle can potentially produce hundreds or thousands

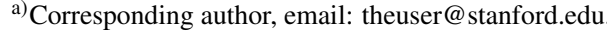

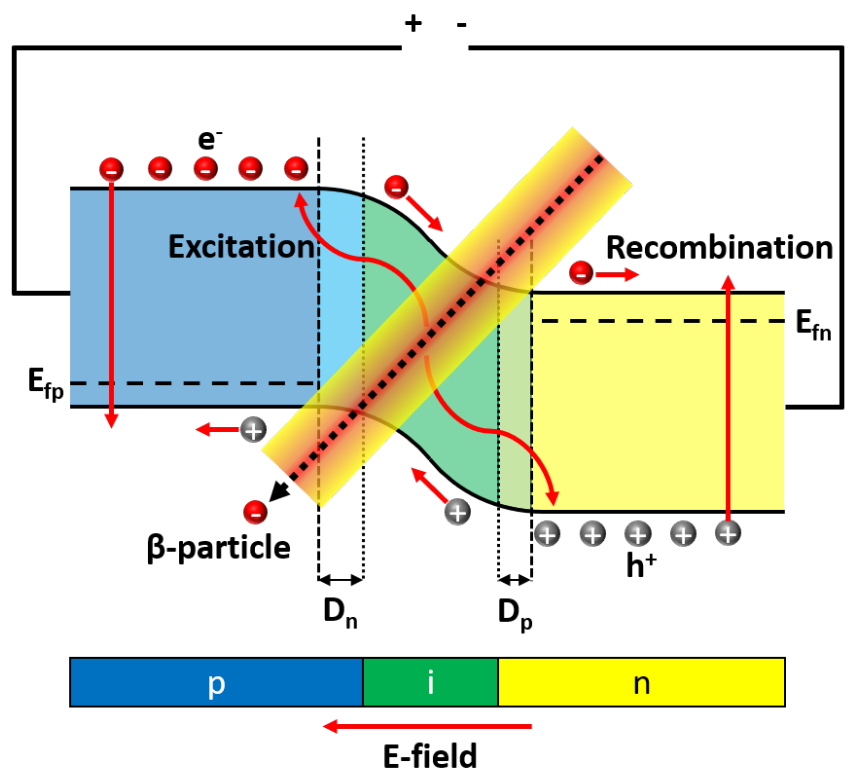

FIG. 1. Band diagram showing charge carrier excitation and recombination as the result of a beta particle strike. Electrons and holes produced in the intrinsic region or one minority carrier diffusion length on either side (represented by $D_{n}$ and $D_{p}$ ) contribute to the generated current. Region widths are not to scale.

of EHPs. The electronic behavior of a p-i-n semiconductor junction subjected to beta radiation can be seen in Fig. 1. Because the energy source for betavoltaic devices is a decaying isotope, radioactive material can be packaged with the device to produce a totally self-contained power source that can function for decades without inputs or maintenance.

The performance of a betavoltaic device depends primarily 


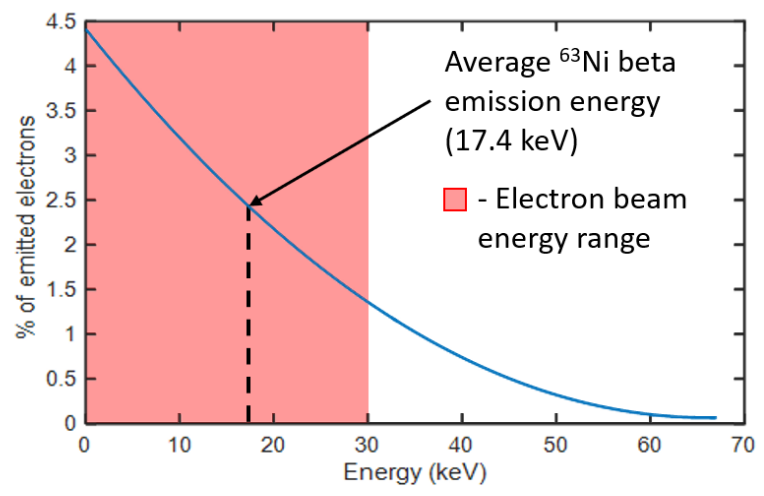

FIG. 2. Beta emission spectrum of ${ }^{63} \mathrm{Ni}$. The curve represents the fraction of electrons emitted at each energy, the dashed line indicates the average energy of the ${ }^{63} \mathrm{Ni}$ spectrum, and the shaded area represents the energy range covered by the electron beam used in this experiment, which had a maximum energy of $30 \mathrm{keV}$.

on three factors: the identity and activity of the radioisotope source, the properties of the semiconductor material, and the geometry of the device ${ }^{617}$ In this study, we investigate the performance of a planar GaN-based betavoltaic device designed to be powered by the isotope ${ }^{63} \mathrm{Ni}$.

${ }^{63} \mathrm{Ni}$ has a number of characteristics that make it ideal for use in betavoltaic devices: it has a relatively long half-life ( $\sim 100$ years), its beta emission spectrum is sufficiently low energy that it does not present a serious radiation safety hazard, and it does not produce alpha particles, gamma radiation or bremsstrahlung x-rays. $\frac{\sqrt{8}}{}$ Moreover, as a metal, it can be incorporated directly into the architecture of a device relatively easily. Properties of isotopes commonly considered for use in betavoltaics can be found in Table I. The ${ }^{63} \mathrm{Ni}$ emission spectrum can be seen in Fig. 2. To allow for easy variation of test parameters, in this study we used an electron beam tuned to an energy of $17.4 \mathrm{keV}$ (the average energy of the ${ }^{63} \mathrm{Ni}$ beta emission spectrum) to replicate the behavior of the isotope in place of the isotope itself.

Gallium nitride $(\mathrm{GaN})$ has several advantages over $\mathrm{Si}$ as the semiconductor material in betavoltaic devices. The higher bandgap ( $3.4 \mathrm{eV}$ vs. $1.12 \mathrm{eV}$ ) increases betavoltaic conversion efficiency, as well as making GaN significantly more robust against radiation-induced damage. ${ }^{9}$ Additionally, GaN can be alloyed with other group III elements, such as aluminum, to further increase the bandgap, and therefore the efficiency 10

The choice of $\mathrm{GaN}$ as a semiconductor material means that (111) Si can, with an appropriate buffer structure, be used as a growth substrate. With a procedure for growing $\mathrm{GaN} \mathrm{p-i-}$ $\mathrm{n}$ structures on $\mathrm{Si}$, it is possible to take advantage of silicon's easy patterning to dramatically increase the surface area of the resulting betavoltaic device without increasing its wafer footprint. A wide variety of techniques exist to modify the geometry of Si substrates to increase surface area; of particular interest for this application is the wet-etching of (001) silicon to produce inverted pyramid or v-groove structures with (111)oriented surfaces, which allow for significant surface area increases while maintaining the (111)-oriented surface needed
TABLE I. Common beta-emitting radioisotopes viable for use in betavoltaic devices

\begin{tabular}{cccc}
\hline \hline Isotope & Mean Energy (keV) & Max Energy (keV) & Half-Life (yrs.) \\
\hline${ }^{3} \mathrm{H}$ & 5.7 & 18.6 & 12.3 \\
${ }^{14} \mathrm{C}$ & 49 & 156 & 5710 \\
${ }^{35} \mathrm{~S}$ & 48.8 & 167.5 & 0.24 \\
${ }^{63} \mathrm{Ni}$ & 17.4 & 66.7 & 100.1 \\
${ }^{90} \mathrm{Sr} /{ }^{90} \mathrm{Y}$ & $195.8 / 933.7$ & $546 / 2280$ & $28.8 / 7.37 \mathrm{e}-3$ \\
${ }^{137} \mathrm{Cs}$ & 188.4 & 1176 & 30.1 \\
${ }^{147} \mathrm{Pm}$ & 62 & 225 & 2.6 \\
\hline \hline
\end{tabular}

for $\mathrm{GaN}$ growth 11112

\section{RADIATION MODELING}

In order to maximize the efficiency of a betavoltaic device, it is important to make the depletion region large to capture as much of the energy from the penetration of beta radiation as possible, without making it so large that charge carriers are no longer able to successfully traverse the junction. p-i-n structures are particularly well-suited for use in betavoltaics due to the fact that their intrinsic region artificially expands the depletion region. This is significant because of the high penetration depth of even low-energy beta particles (several microns) compared to the thickness of the natural depletion region in typical GaN homojunctions $(<100 \mathrm{~nm}) !^{\frac{13] 14}{10}}$ To verify the beta particle penetration and energy deposition depths for matching with the depletion width, modeling of radiation penetration and energy deposition in $\mathrm{GaN}$ was done using the Monte Carlo particle physics simulation platform CASINO $\underline{15}$

Two simulation sets were run: one subjected to an isotropic particle source producing the full ${ }^{63} \mathrm{Ni}$ beta emission spectrum to simulate the behavior of the actual ${ }^{63} \mathrm{Ni}$ isotope, and one subjected to an electron beam source tuned to $17.4 \mathrm{keV}$ (the average energy of the ${ }^{63} \mathrm{Ni}$ spectrum) to simulate the electron beam used in this experiment. Both simulations were designed such that the incident particle count $n=50,000$. These simulations show that the vast majority $(\sim 90 \%)$ of the energy deposited by ${ }^{63} \mathrm{Ni}$ beta radiation is deposited within or near the i-GaN layer, which means that it is deposited in the depletion region. Results of these simulations can be seen in Fig. 3. In addition to the fact that these two beta sources have different energies (fixed energy at $17.4 \mathrm{keV}$ vs. spectrum with average energy of $17.4 \mathrm{keV}$ ), they also differ in angle of incidence. The beam source is oriented perpendicular to the surface, while particles produced by the spectrum source, an isotropic emitter, can impinge upon the GaN surface at any angle between $0^{\circ}$ and $90^{\circ}$. Particles entering at shallower angles do not penetrate as deeply into film, shifting the net energy deposition much closer to the material surface than would be expected from an orthogonal beam. Results of the simulated beta particle penetration were used to select the thicknesses of the layers in the fabricated device, in order to capture as much energy as possible within the depletion region. 

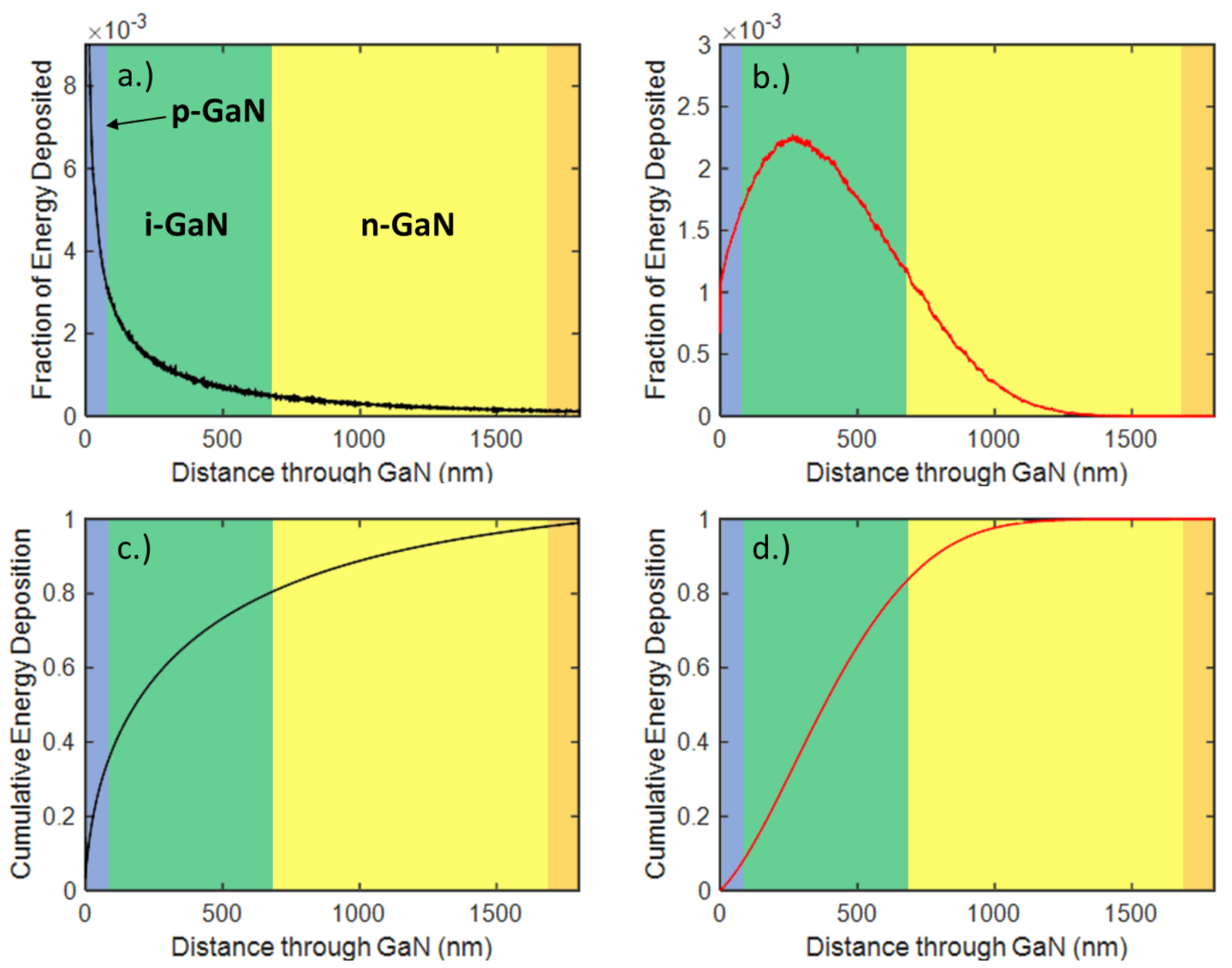

FIG. 3. CASINO simulation results for beta particle penetration into GaN, with layer thicknesses shown. a.) and b.) show the energy deposition as a function of depth through GaN, whereas c.) and d.) display the cumulative fraction of the total energy deposited as a function of depth. a.) and c.) (in black) show results from a simulation using a source representing the full ${ }^{63} \mathrm{Ni}$ beta emission spectrum, while the simulation represented in b.) and d.) (in red) used a beam source oriented perpendicular to the surface tuned to an energy of $17.4 \mathrm{keV}$.

\section{FILM GROWTH AND DEVICE FABRICATION}

Device fabrication began with deposition of a 5-layered AlN/AlGaN/GaN buffer structure on an RCA-cleaned 4-inch (111) silicon wafer using metalorganic chemical vapor deposition (MOCVD), followed by MOCVD growth of a 3-layered $\mathrm{GaN}$ p-i-n structure with a $2 \mathrm{~nm} \mathrm{GaN}$ cap to passivate the active layers during processing. The purpose of the buffer structure is to step smoothly from the lattice constant of (111) $\mathrm{Si}$ $(3.84 \AA)$ to the lattice constant of $\mathrm{GaN}(3.19 \AA)$ to reduce film strain and minimize the formation of dislocations during growth. The entire stack, including the p-i-n structure, was produced in a single MOCVD process step without breaking vacuum to minimize contamination and improve lattice matching between the films. The growth details of this buffer structure have been described previously 16 To create the nand p-type regions, the GaN was doped during growth with silicon and magnesium, respectively. Because GaN has a natural tendency towards unintentional n-type doping as result of the donor-like behavior, the i-region was lightly counterdoped with Mg. Based on the simulation results and comparison with existing literature, the $\mathrm{p}-\mathrm{GaN}$ region had a thickness of $80 \mathrm{~nm}$ with a dopant concentration of $4 \times 10^{17} \mathrm{~cm}^{-3}$, the i-GaN region had a thickness of $600 \mathrm{~nm}$ with a dopant concentration of $1 \times 10^{15} \mathrm{~cm}^{-3}$, and the $\mathrm{n}-\mathrm{GaN}$ region had a thickness of 80 $\mathrm{nm}$ with a dopant concentration of $3 \times 10^{18} \mathrm{~cm}^{-3}$ !17/18

The mesa etch was accomplished through a novel triple hard mask etch technique, in which three different films are deposited and patterned sequentially in order to act as hard masks for successive etches into the $\mathrm{GaN} \mathrm{p}$-i-n structure. First, a $100 \mathrm{~nm}$ thick $\mathrm{SiO}_{2}$ film was deposited on the $\mathrm{GaN}$ buffer structure using plasma-enhanced chemical vapor deposition (PECVD). Then, this film was patterned to expose the areas of the p-GaN layer that were to be etched. Next, a $100 \mathrm{~nm}$ chromium film was evaporated onto the surface and patterned to expose the areas of the i-GaN that were to be etched. Last, a $100 \mathrm{~nm}$ nickel film was evaporated onto the surface and patterned to expose the areas of the $n-G a N$ that were to be etched. 

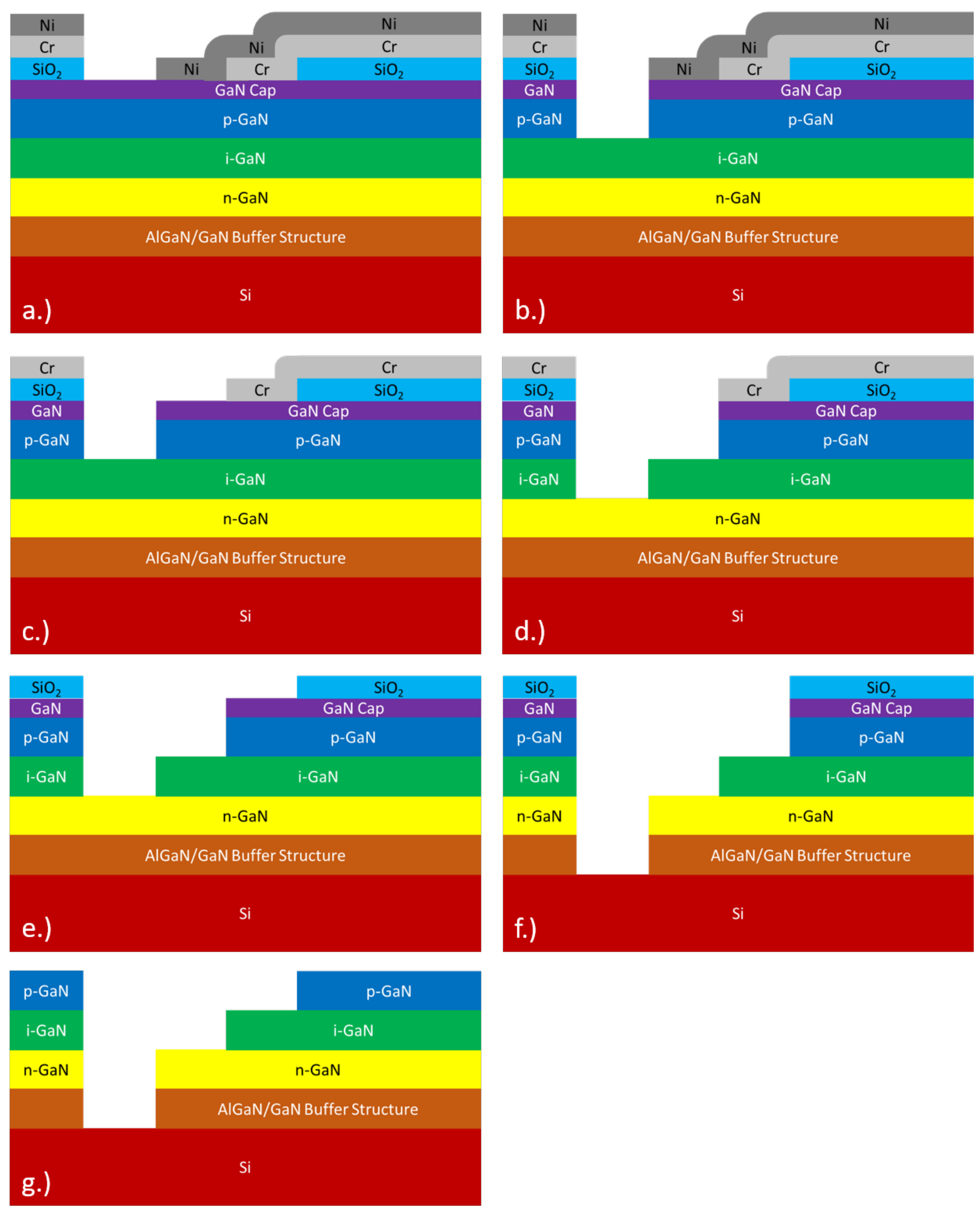

FIG. 4. Triple mesa etch process steps: a.) Full $\mathrm{AlGaN} / \mathrm{GaN}$ film stack with $\mathrm{SiO}_{2}, \mathrm{Cr}$, and Ni hard masks deposited and patterned. b.) Dry etch for n-GaN patterning. c.) Wet etch to strip Ni hard mask. d.) Dry etch for i-GaN patterning. e.) Wet etch to strip Cr hard mask. f.) Dry etch for $\mathrm{p}-\mathrm{GaN}$ patterning. g.) Final $\mathrm{AlGaN} / \mathrm{GaN}$ architecture after removal of $\mathrm{SiO}_{2}$ hard mask and $\mathrm{GaN}$ cap. 

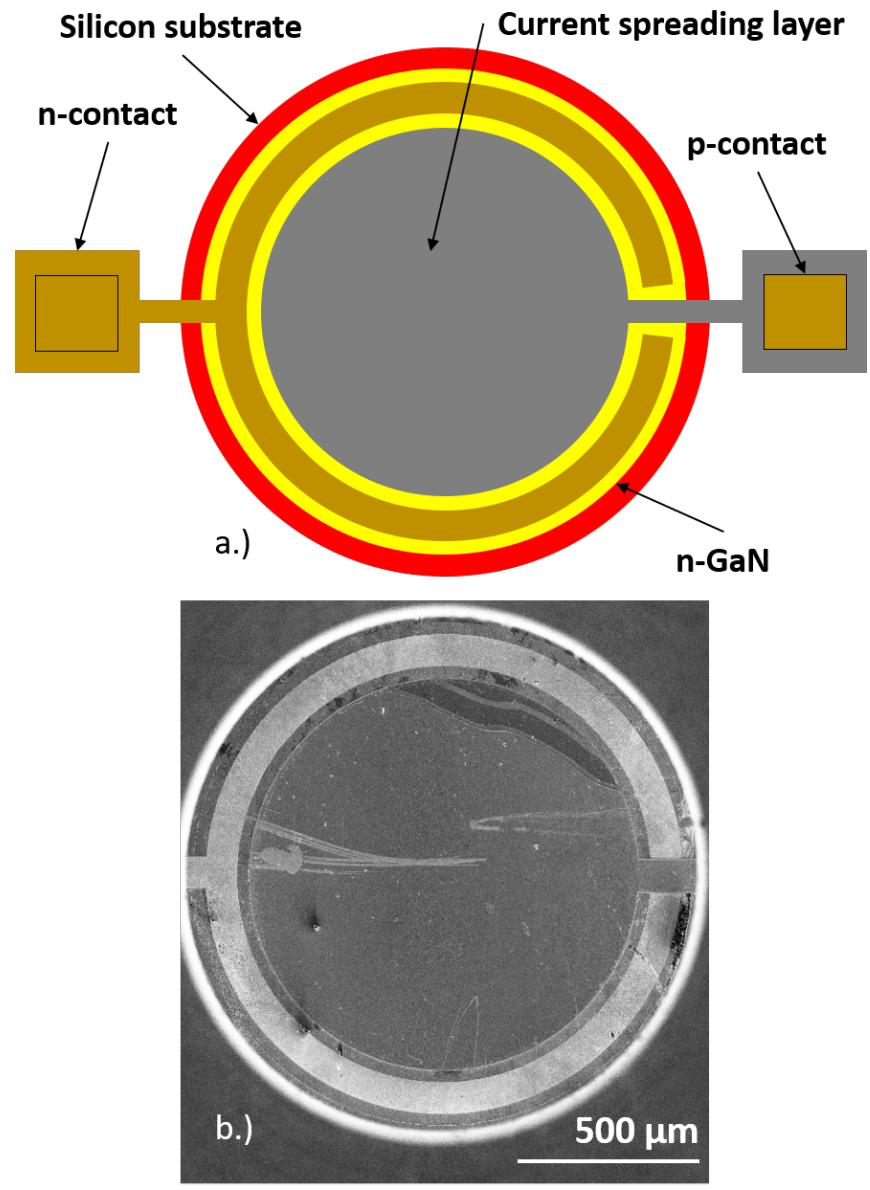

FIG. 5. a.) Top-down schematic of betavoltaic device architecture and b.) Top-down SEM of fabricated betavoltaic device.

After the three hard masks were deposited, a series of alternating dry and wet etches were done to sequentially etch layers of $\mathrm{GaN}$ and strip away the hard masks: First, a dry etch was done to pattern the $\mathrm{p}-\mathrm{GaN}$, then the nickel mask was chemically removed using a metal-selective wet etch (Transene Nickel Etchant TFG, $40^{\circ} \mathrm{C}, 10 \mathrm{~min}$ ). Second, another dry etch was done to pattern the i-GaN, then the chromium mask was removed using another metal-selective wet etch (Transene Chromium Etchant $1020 \mathrm{AC}, 40^{\circ} \mathrm{C}, 10$ min.). Third, a dry etch was used to pattern the $\mathrm{n}-\mathrm{GaN}$, forming the isolation trenches around each device, after which the $\mathrm{SiO}_{2}$ layer was chemically stripped in a $2 \% \mathrm{HF}$ solution. Finally, a very brief dry etch was done to strip the GaN cap and expose the $n-G a N$ surface.

After the triple mesa etch, Ohmic contacts were deposited on the p- and n-type GaN layers using three separate metal evaporation and liftoff steps. The first step deposited a 8/10 $\mathrm{nm} \mathrm{Ni} / \mathrm{Au}$ (post metallization rapid thermal anneal: 470 ${ }^{\circ} \mathrm{C}, 120 \mathrm{~s}, 2: 1 \mathrm{~N}_{2} / \mathrm{O}_{2}$, ambient pressure) current-spreading layer to facilitate the collection of charge carriers from the p-GaN layer. The second step deposited 20/100/40/80 nm $\mathrm{Ti} / \mathrm{Al} / \mathrm{Pt} / \mathrm{Au}$ multilayer $\mathrm{n}-\mathrm{GaN}$ contacts. The third step deposited the gold bond pads used to passivate the metal stacks

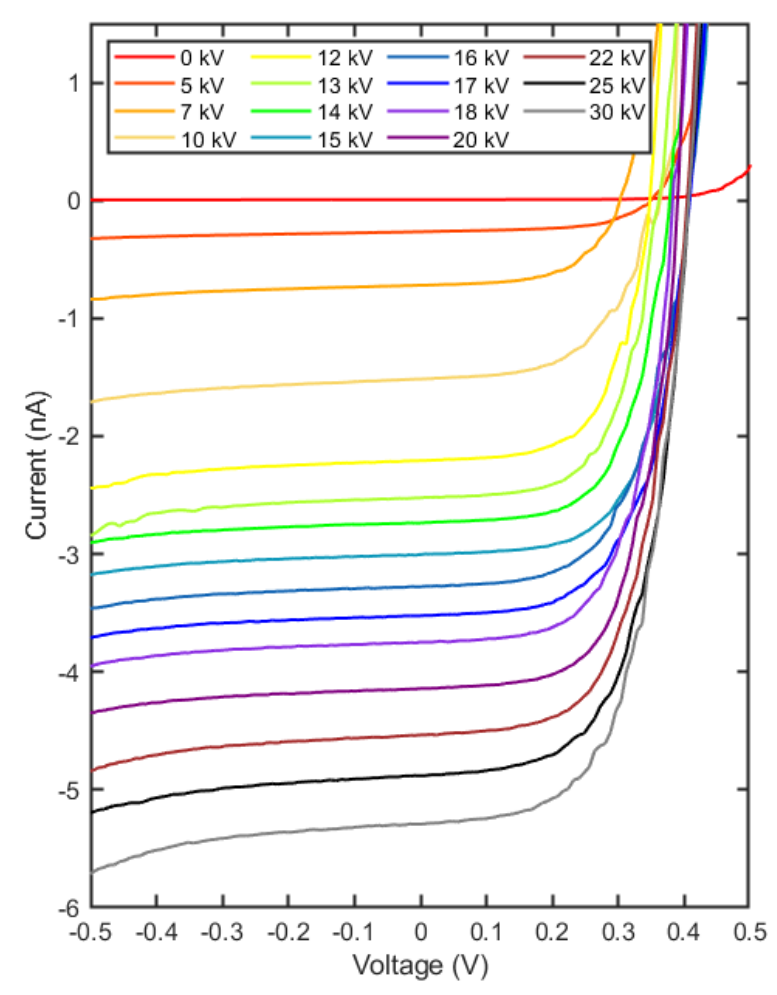

FIG. 6. Betavoltaic I-V measurements under $26 \mathrm{nA}$ electron beam irradiation over an accelerating voltage range of $0-30 \mathrm{kV}$.

and allow for electrical contact with the device via wirebonding. Top down schematic and SEM can be seen in Fig. 5

\section{ELECTRON BEAM IRRADIATION}

To test the devices in a radiation environment, they were subjected to electron beam irradiation from a FEI Magellan 400 XHR SEM with the accelerating voltage tuned between 0 and $30 \mathrm{kV}$ and the current fixed at $26 \mathrm{nA}$. In order to conduct in-situ electrical measurements during irradiation, the devices were wirebonded to a printed circuit board (PCB) that was connected to an external measurement setup through electrical feedthrough ports built into the SEM chamber. A rectangular $995 \mu \mathrm{m} \times 860 \mu \mathrm{m}$ window at the center of the device under test was continuously irradiated during I-V measurements, resulting in an electron flux density of $0.19 \mathrm{~nm}^{-2}$ or $3.04 \mu \mathrm{A} / \mathrm{cm}^{2}$. The irradiation area was scanned at $88 \mathrm{~Hz}$ to eliminate the influence of any position-specific device defects on the measured device current. The I-V curves generated by these measurements can be found in Fig. 6 

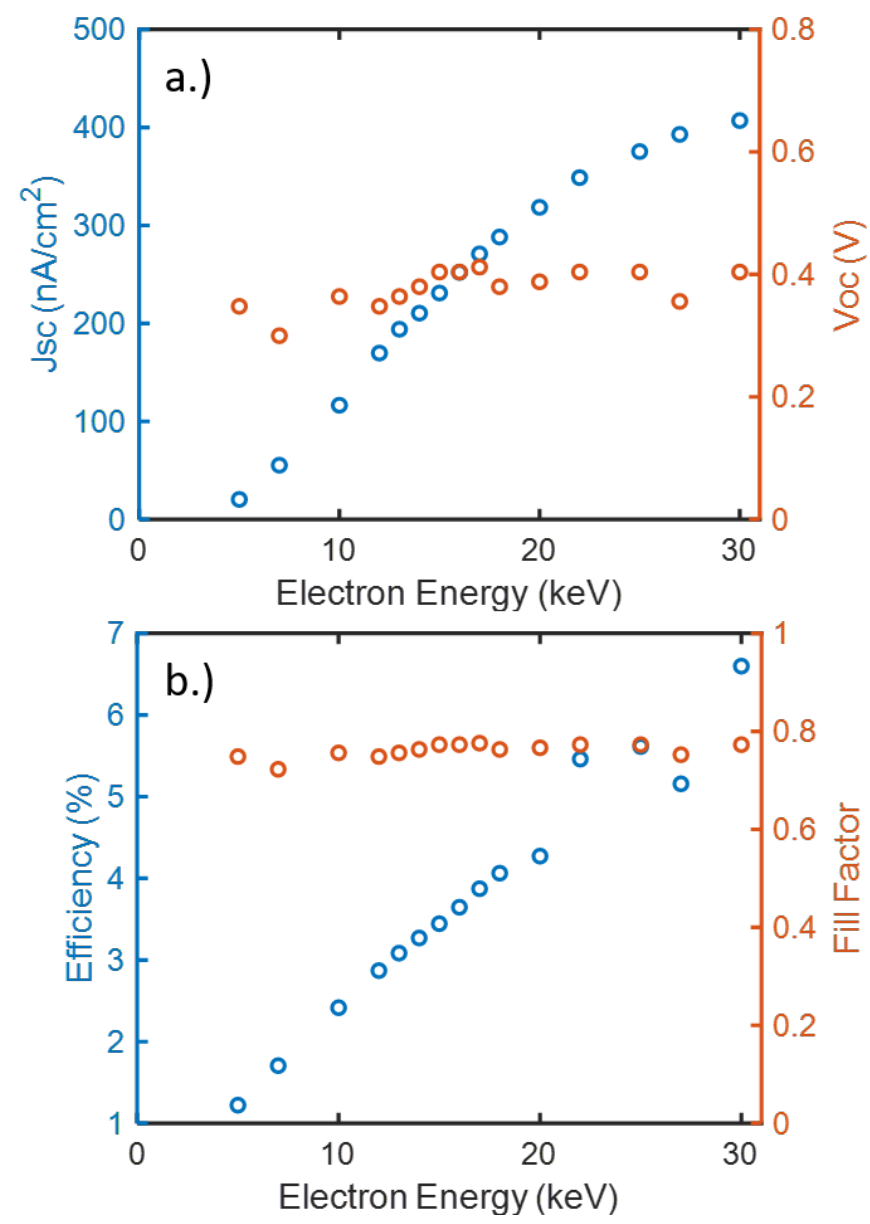

FIG. 7. Betavoltaic device parameters as a function of incident electron beam energy. a.) displays short-circuit current density and opencircuit voltage, and b.) displays overall device efficiency and fill factor.

\section{RESULTS AND DISCUSSION}

The total device efficiencies were calculated using eqs. (13 ) and the fill factor (FF) was calculated using eqs. (4-5), where $\eta$ is overall efficiency, $P_{\text {out }}$ is the power output of the betavoltaic device, $P_{i n}$ is the power of the electron beam, $I_{m}$ and $V_{m}$ are the current and voltage at the point of maximum power, $I_{\text {beam }}$ and $V_{\text {beam }}$ are the current and voltage of the incident electron beam, and $V_{o c}$ is the open circuit voltage of the betavoltaic device. $20 \mid 21$

$$
\begin{gathered}
\eta=\frac{P_{\text {out }}}{P_{\text {in }}} \\
P_{\text {out }}=I_{m} * V_{m} \\
P_{\text {in }}=I_{\text {beam }} * V_{\text {beam }}
\end{gathered}
$$

$$
\begin{gathered}
v_{O C}=\frac{V_{O C}}{k_{B} T} \\
F F=\left[v_{O C}-\ln \left(v_{O C}+0.72\right)\right] /\left(v_{O C}+1\right)
\end{gathered}
$$

Fig. 7 contains two plots displaying parameters of the device at different e-beam accelerating voltages.

Analysis of the I-V measurements taken under irradiation indicate a maximum efficiency of $6.6 \%$ and FF of 0.77 . As the incident beam energy is increased, the device efficiency and the short-circuit current increase, and the $V_{o c}$ and FF, which do not depend on incident energy, remain relatively constant. The continued increases of $\eta$ and $J_{s c}$ occur because the overall amount of energy being introduced into the betavoltaic is increasing, while the incident beam energy is still within the range of the ${ }^{63} \mathrm{Ni}$ beta emission spectrum that device was designed to effectively absorb. Although differences in device architecture, radiation source, and test methodology make direct comparison difficult, these values are comparable to those that have been reported previously for similar devices.

To the best of the authors' knowledge, at the time of publication, this work represents the highest experimentally demonstrated conversion efficiency and fill factor for a gallium nitride-based betavoltaic device. A comparison of the results of this work (overall conversion efficiency and fill factor) with previous results of $\mathrm{GaN}$-based betavoltaic devices from literature can be found in Fig 8 .

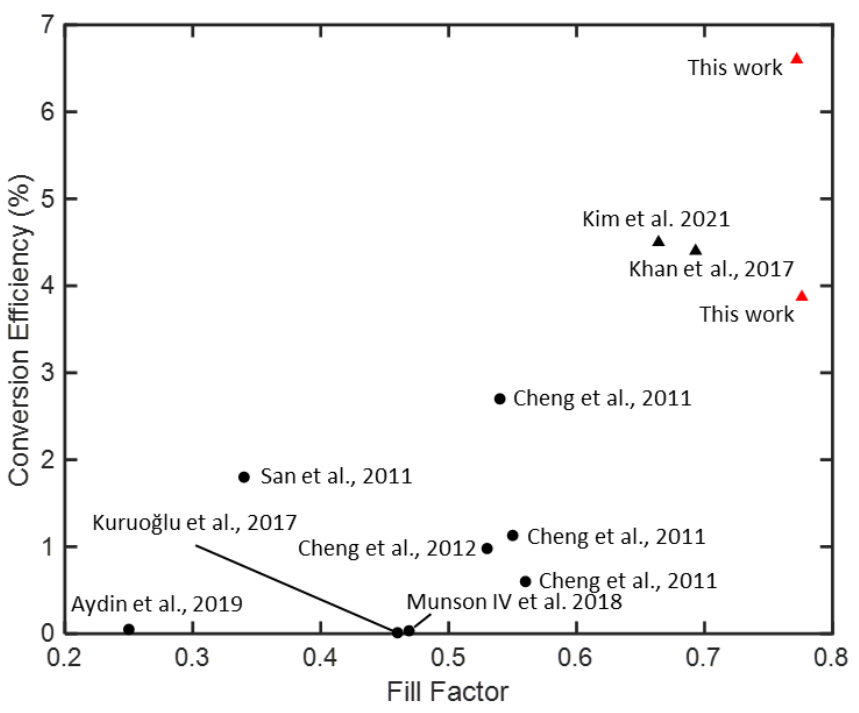

FIG. 8. Comparison of results from this work with results from previously published works on $\mathrm{GaN}$-based betavoltaic devices. Circles represent devices irradiated with ${ }^{63} \mathrm{Ni}$ sources, triangles represent devices irradiated with electron beams.

There are a number of possible ways to optimize the device to further improve performance, in addition to growing on textured silicon substrates. One potential method includes 
incorporating ${ }^{63} \mathrm{Ni}$ directly into the $\mathrm{p}-\mathrm{GaN}$ contact to bring the isotope source directly into contact with the device surface, eliminating losses from electrons reflected or absorbed by the current spreading layer. Additionally, further optimizing the film thicknesses (as well as the film quality) could allow for effective capture of a higher fraction of the energy deposited by incident beta radiation.

\section{CONCLUSIONS}

In this work, we demonstrate fabrication of a p-i-n GaN-onSi betavoltaic energy converter fabricated using a triple-hardmask mesa etch process. Device design was informed by beta particle penetration simulations generated using Monte Carlo simulation platform CASINO. Initial measurements indicate a maximum efficiency of $6.6 \%$, fill factor of 0.77 , open-circuit voltage of $412 \mathrm{mV}$, and short-circuit current of $407 \mathrm{nA} / \mathrm{cm}^{2}$. This conversion efficiency and fill factor are the highest experimentally demonstrated in gallium nitride to date. It is expected that the device performance could be further improved by growing the $\mathrm{AlGaN} / \mathrm{GaN}$ film stack on textured $\mathrm{Si}$ substrates to increase the effective surface area, by optimizing the device layer thicknesses, and by integrating ${ }^{63} \mathrm{Ni}$ directly into the $\mathrm{p}-\mathrm{GaN}$ current-spreading layer so that it is in direct contact with the device surface.

\section{ACKNOWLEDGMENTS}

Fabrication and material characterization work were performed in part at the Stanford Nanofabrication Facility (SNF) and Stanford Nano Shared Facilities (SNSF). This material is based upon work supported by the National Science Foundation Graduate Research Fellowship [Grant No.DGE 1147470]. M.B. would like to acknowledge the Stanford Nano Shared Facilities (SNSF) with NSF support (ECCS-1542152) and NSF Graduate Fellowship (DGE-1656518).

\footnotetext{
${ }^{1}$ R. Paul, "Radioactive battery employing intrinsic semiconductor," (1956), uS Patent 2,745,973.

${ }^{2}$ L. C. Olsen, S. E. Seeman, B. I. Griffia, and C. J. Ambrose, "Nuclear battery," (1972), uS Patent 3,706,893.

${ }^{3}$ C. Honsberg, W. A. Doolittle, M. Allen, and C. Wang, "Gan betavoltaic energy converters," in Conference Record of the Thirty-first IEEE Photovoltaic Specialists Conference, 2005. (IEEE, 2005) pp. 102-105.

${ }^{4}$ G. Park, T. Rosing, M. D. Todd, C. R. Farrar, and W. Hodgkiss, "Energy harvesting for structural health monitoring sensor networks," Journal of Infrastructure Systems 14, 64-79 (2008).

${ }^{5}$ C. A. Klein, "Bandgap dependence and related features of radiation ionization energies in semiconductors," Journal of Applied Physics 39, 20292038 (1968).

${ }^{6}$ L. C. Olsen, "Review of betavoltaic energy conversion," in NASA Conference Publication (NASA, 1993) pp. 256-256.

${ }^{7}$ A. Sachenko, A. Shkrebtii, R. Korkishko, V. Kostylyov, M. Kulish, and I. Sokolovskyi, "Efficiency analysis of betavoltaic elements," Solid-State Electronics 111, 147-152 (2015).

${ }^{8}$ C. Poletiko, "Determination of nickel-63," Environment international 14, 387-390 (1988).
}

${ }^{9}$ K.-a. Son, A. Liao, G. Lung, M. Gallegos, T. Hatake, R. D. Harris, L. Z. Scheick, and W. D. Smythe, "Gan-based high temperature and radiationhard electronics for harsh environments," Nanoscience and Nanotechnology Letters 2, 89-95 (2010).

${ }^{10}$ N. Nepal, J. Li, M. Nakarmi, J. Lin, and H. Jiang, "Temperature and compositional dependence of the energy band gap of algan alloys," Applied Physics Letters 87, 242104 (2005).

${ }^{11}$ Z. Cheng, Z. Zhao, H. San, and X. Chen, "Demonstration of a gan betavoltaic microbattery," in 2011 6th IEEE International Conference on Nano/Micro Engineered and Molecular Systems (IEEE, 2011) pp. 10361039.

${ }^{12}$ K. E. Bower, Y. A. Barbanel, Y. G. Shreter, and G. W. Bohnert, Polymers, phosphors, and voltaics for radioisotope microbatteries (CRC press, 2002).

${ }^{13}$ P. Kozodoy, S. P. DenBaars, and U. K. Mishra, "Depletion region effects in mg-doped gan,” Journal of Applied Physics 87, 770-775 (2000).

${ }^{14}$ M. Foussekis, A. Baski, and M. A. Reshchikov, "Photoadsorption and photodesorption for gan," Applied Physics Letters 94, 162116 (2009).

${ }^{15} \mathrm{O}$. Kurniawan and V. Ong, "Investigation of range-energy relationships for low-energy electron beams in silicon and gallium nitride," Scanning: The Journal of Scanning Microscopies 29, 280-286 (2007).

${ }^{16}$ X. Xu, J. Zhong, H. So, A. Norvilas, C. Sommerhalter, D. G. Senesky, and M. Tang, "Wafer-level mocvd growth of algan/gan-on-si hemt structures with ultra-high room temperature 2deg mobility," AIP Advances 6, 115016 (2016).

${ }^{17}$ H. Cho, C. Kim, and C.-H. Hong, "Electron capture behaviors of deep level traps in unintentionally doped and intentionally doped n-type gan,' Journal of applied physics 94, 1485-1489 (2003).

${ }^{18}$ T. Zhu and R. A. Oliver, "Unintentional doping in gan," Physical Chemistry Chemical Physics 14, 9558-9573 (2012).

${ }^{19}$ Z. Cheng, X. Chen, H. San, Z. Feng, and B. Liu, "A high open-circuit voltage gallium nitride betavoltaic microbattery," Journal of Micromechanics and Microengineering 22, 074011 (2012).

${ }^{20}$ M. R. Khan, J. R. Smith, R. P. Tompkins, S. Kelley, M. Litz, J. Russo, J. Leathersich, F. S. Shahedipour-Sandvik, K. A. Jones, and A. Iliadis, "Design and characterization of gan pin diodes for betavoltaic devices," Solid-State Electronics 136, 24-29 (2017).

${ }^{21}$ A. Sachenko, A. Shkrebtii, R. Korkishko, V. Kostylyov, M. Kulish, I. Sokolovskyi, and M. Evstigneev, "Analysis of the attainable efficiency of a direct-bandgap betavoltaic element," Journal of Physics D: Applied Physics 48, 455101 (2015).

${ }^{22}$ P. Kozodoy, H. Xing, S. P. DenBaars, U. K. Mishra, A. Saxler, R. Perrin, S. Elhamri, and W. Mitchel, "Heavy doping effects in mg-doped gan," Journal of Applied Physics 87, 1832-1835 (2000).

${ }^{23}$ C. Zai-Jun, S. Hai-Sheng, C. Xu-Yuan, L. Bo, and F. Zhi-Hong, "Demonstration of a high open-circuit voltage gan betavoltaic microbattery," Chinese Physics Letters 28, 078401 (2011).

${ }^{24}$ Z. Cheng, H. San, Z. Feng, B. Liu, and X. Chen, "High open-circuit voltage betavoltaic cell based on gan pin homojunction," Electronics letters 47, 720-722 (2011)

${ }^{25} \mathrm{~S}$. Aydin and E. Kam, "Investigation of nickel-63 radioisotope-powered gan betavoltaic nuclear battery," International Journal of Energy Research 43, 8725-8738 (2019).

${ }^{26}$ N. A. Kuruoğlu, O. Özdemir, and K. Bozkurt, "Betavoltaic study of a gan pin structure grown by metal-organic vapour phase epitaxy with a ni-63 source," Thin Solid Films 636, 746-750 (2017).

${ }^{27}$ C. Munson, Q. Gaimard, K. Merghem, S. Sundaram, D. Rogers, J. De Sanoit, P. L. Voss, A. Ramdane, J. P. Salvestrini, and A. Ougazzaden, "Modeling, design, fabrication and experimentation of a gan-based, 63ni betavoltaic battery," Journal of Physics D: Applied Physics 51, 035101 (2017).

${ }^{28}$ D.-S. Kim, Y. J. Yoon, J. S. Lee, I. M. Kang, and J.-H. Lee, "Experimental and simulation study of power performance improvement of gan pin betavoltaic cell,' International Journal of Energy Research (2021). 